\title{
Variability of PT-INR values measured by point of care devices INRatio/INRatio 2 and CoaguChek XS and standard laboratory method. A cross-sectional study
}

\author{
Chikayoshi Komatsu', Harukazu Iseki², Shinichi Goto², Shinya Goto²
}

\author{
${ }^{1}$ Komatsu Cardiovascular Clinic, Hadano, Japan \\ 2Department of Medicine (Cardiology), School of Medicine, Tokai University, Isehara, \\ Japan
}

Submitted: 9 July 2020

Accepted: 17 August 2020

Arch Med Sci

DOI: https://doi.org/10.5114/aoms.2020.99165

Copyright @ 2020 Termedia \& Banach

\section{Abstract}

Introduction: Various point-of-care (POC) devices are available for measurement of PT-INR with whole blood in patients receiving vitamin $\mathrm{K}$ antagonists (VKAs). However, the variability in values measured with various POC devices and traditional plasma-based laboratory measurement in clinical practice has yet to be clarified.

Material and methods: PT-INR values measured with the same blood sample by POC devices of INRatio/INRatio 2 (INRatio) and the CoaguChek XS portable INR monitor (CoaguChek) were compared with those measured with traditional plasma-based laboratory measurement (lab-INR) in 1,347 measurements from 393 patients. The difference between the PT-INR values obtained from Lab-INR and POC devicees were expressed as $\Delta$. To quantify the relationships among values obtained by various methods, regression analysis and the Bland-Altman method were applied.

Results: PT-INR values measured with INRatio were higher than lab-INR with $\Delta=-0.33 \pm 0.38$ (mean \pm SD; $95 \% \mathrm{Cl}:-0.31--0.35$ ). Pearson's correlation coefficient between the values measured with INRatio and lab-INR was 0.784 . The PT-INR values measured with CoaguChek were not different from lab-INR with $\Delta-0.08 \pm 0.15$ (mean \pm SD; $95 \% \mathrm{Cl}$ : $-0.07--0.09$ ). The correlation between the value obtained with CoaguChek XS and lab PT-INR was high with Pearson's correlation coefficient 0.951.

Conclusions: Even with the blood obtained from the same patients treated with the same dose of warfarin, the PT-INR values measured with INRatio were higher than traditional laboratory measurements. The apparent "quality of warfarin control" is influenced by the methods of PT-INR measurements.

Key words: point-of-care testing, anticoagulation, PT-INR, atrial fibrillation, vitamin $\mathrm{K}$ antagonists.

\section{Introduction}

Vitamin K antagonists (VKAs) were widely used until various, so-called, direct oral anticoagulants (DOACs) became popularized [1]. Despite more limited use than before [2, 3], VKAs are still the standard of care in atrial fibrillation (AF) patients with hemodynamically overt mitral stenosis and a mechanical heart valve. PT-INR measurements are necessary to achieve the best balance between antithrombotic effects and the risk of serious

\author{
Corresponding author: \\ Shinya Goto, MD, PhD \\ Department of Medicine \\ (Cardiology) \\ School of Medicine \\ Tokai University \\ 143 Shimokasuya \\ Isehara 259-1193 \\ Japan \\ Phone: +81 463-93-1121 \\ Fax: +81 463+93-6679 \\ E-mail: sgoto3@mac.com
}


bleeding complications upon using VKAs. Practice guidelines recommend PT-INR 2-3 for the vast majority of patients on the glove $[4,5]$. But, a lower target PT-INR of 1.6-2.6 is recommended in elderly Japanese patients [6]. PT-INR in nature is neither accurate nor convenient [7-9]. Separation of plasma from whole blood and transfer to a central laboratory take considerable time. Several pointof-care (POC) devices enabling the measurement of PT-INR with whole blood have been developed $[10,11]$. However, values obtained by POC devices and traditional laboratory measurements have not been compared widely in real world clinical practice. Target PT-INR using a specific POC device may differ substantially from the values recommended using lab-INR.

Several POC devices have been used in warfarin arms of clinical trials developing DOACs in patients with non-valvular atrial fibrillation (AF) [12-14]. The POC devices were necessary in these clinical trials because the trials were conducted in double blinded fashion. Neither physicians nor patients knew whether the patients were treated with warfarin or the DOAC. The number of tablets (either warfarin or placebo) should be increased/decreased based on the values that appeared on the POC devices during the trial. The POC devices used in these trials should have a function to show the measured values in the warfarin arm but provide dummy values in the DOAC arm. In the four of the DOAC developmental trials in stroke prevention in AF, target PT-INR was established as 2-3 assuming there are no differences among values obtained by each POC devices and lab-INR [12-15]. All 4 DOAC development trials demonstrated that the quality of warfarin controls are high enough, meaning the time in the therapeutic range (TTR) in warfarin arm was more than $60 \%$. However, the apparent quality of warfarin therapy may be influenced by the accuracy of the POC device used in each trial. Indeed, the accuracy of PT-INR measurements with POC devices is reported not to be high enough to replace lab-INR [16]. Moreover, the US Food and Drug Administration (FDA) issued a medical device recall notice for the Alere INRatio Monitor System (formally known as the HemoSense INRatio device: INRatio) because the values obtained from this whole blood POC device become low in anemic condition as compared to the values obtained by traditional plasma-based lab measurements [17]. Thus, we proposed the clinical hypothesis that the PT-INR values obtained with POC devices may differ from those measured with lab-INR in a real world setting.

Here we report the difference among values of PT-INR obtained from 2 of the POC devices (INRatio/INRatio 2, CoaguChek XS) with traditional plasma-based laboratory measurement (lab-INR).

\section{Material and methods}

\section{Patient population}

This is a single-center, retrospective analysis of prothrombin time (PT)-international normalized ratio (INR) measurements in patients treated with the VKA warfarin. The study was approved by the ethics committee of Komatsu Cardiovascular Clinic. According to local regulatory requirements, the approved protocol was posted in the clinic where every patient was able to see it. The study was conducted as a hypothesis generation study with no pre-determined sample size. Two of the POC devices, namely INRatio/INRatio 2 (Alere, San Diego, US) and CoaguChek XS (Roche, Switzerland), were selected as testing POCs due to availability to compare with traditional plasma-based laboratory measurements in 3 phases of 2011-2012, 2015, and 2016 of consecutive patients.

\section{Blood sampling and PT-INR measurements}

Blood sampling was conducted by standard venipuncture. For measurements of PT-INR with POC devices (INRatio/INRatio 2 and CoaguChek XS), collected blood was directly applied to the devices with the standard procedure. For laboratory measurements, collected blood was anticoagulated with $3.2 \%$ sodium citrate. Then, the samples were centrifuged at $1,500 \mathrm{~g}$ for $10 \mathrm{~min}$ to separate plas$\mathrm{ma}$, and then sent to the testing center (SYSMEX, Co, Ltd, Tokyo, Japan).

PT-INR was measured using POC devices of INRatio/INRatio 2 and CoaguChek XS at the clinic. The values were represented as INRatio and CoaguChek, respectively. For traditional plasma-based measurements, PT-INR measurements were conducted with a full-auto analyzer CA7000 (Sysmex Corporation, Kobe, Japan), and CS5100 (Sysmex Corporation, Kobe, Japan) at the central laboratory. Tissue factor Thromborel S (ISI 0.94-1.02, Sysmex Corporation, Kobe, Japan) was used as the thromboplastin agent. The measured values were represented as lab-INR.

\section{Statistical analysis}

All measured values were expressed as mean \pm SD unless otherwise specified. The difference between the values measured by POC devices and lab-INR were shown as $\Delta=$ (values measured by regular laboratory data) - (values measured by POC). The $\Delta$ was also shown as a Bland-Altman plot where the $x$-axis represent mean PT-INR values measured with both lab-INR and by the POC device. The $\Delta$ values were shown with the $95 \%$ confidential interval. The $\Delta$ was assumed as statically high/low when the $95 \% \mathrm{Cl}$ did not cross each other. Pearson's correlation coefficients were cal- 
culated to quantify the relationship between labINR and POC measured values.

\section{Results}

\section{Patient population}

In total 393 patients were included in this study. As shown in Table I, warfarin was used for stroke prevention in non-valvular atrial fibrillation (AF). The major comorbidities in the target patient population were hypertension (143 patients), type 2 diabetes mellitus ( 52 patients), chronic kidney disease (32 patients), and ischemic heart disease (31 patients). The mean $\mathrm{CHADS}_{2}$ score of patients was 2.0.

\section{PT-INR values measured with traditional plasma-based laboratory measurements (lab-INR) and those measured by INRatio/ INRatio 2 and CoaguChek XS}

The total number of PT-INR measurements was 1,347 . The vast majority of measurements were multiple measurements during the course of our study from the same patients as shown in Table II. There were no apparent trends among PT-INR values and number of replicated measurements. The comparison between INRatio and lab-INR were available in 1,342 measurements. Figure $1 \mathrm{~A}$ shows the distribution of $-\Delta$, which represents the values obtained by Lab-INR - those obtained by INRatio. It is of note that the values differ substantially when PT-INR values are 2.5 or higher. As shown in Figure $1 \mathrm{~B}$, the values obtained by INRatio had a correlation of 0.7839 with lab-INR. PT-INR values measured as INRatio were higher than Lab-INR with $\Delta$ of $-0.33 \pm 0.38$ (mean \pm SD, $95 \% \mathrm{Cl}$ : $-0.31--0.35)$.
Table I. Baseline characteristics

\begin{tabular}{|lc|}
\hline Characteristics & $\begin{array}{c}\text { Number } \\
\text { in target } \\
\text { population }\end{array}$ \\
\hline Female & 37.5 \\
\hline Age [years] & $74.3 \pm 8.9$ \\
\hline \begin{tabular}{l} 
Reason for warfarin use: \\
\hline Non-valvular AF
\end{tabular} & $94.5 \%$ \\
\hline Mitral or aortic valvular replacement & $3.0 \%$ \\
\hline Ox of venous thromboembolism & $1.0 \%$ \\
\hline Hypertension & $1.0 \%$ \\
\hline Type 2 diabetes mellitus & $70.8 \%$ \\
\hline Ischemic cardiovascular diseases & $25.7 \%$ \\
\hline
\end{tabular}

Figure $2 \mathrm{~A}$ shows the relationship between the values obtained by CoaguChek XS and lab-INR in 1,345 measurements. There was no difference between the value measured by CoaguChek XS and lab-INR as measured with $\Delta$ of $-0.08 \pm 0.16$ (mean $\pm \mathrm{SD}, 95 \% \mathrm{Cl}:-0.07-0.09)$. As shown in Figure 2 $B$, there was a strong positive correlation between PT-INR measured by CoaguChek XS and lab-INR with correlation coefficient of 0.9508 .

\section{Discussion}

PT-INR measurements are widely used for controlling anticoagulation therapy with the use of VKAs [12-15]. Laboratory measurement of PT-INR with a plasma sample is an established standard

Table II. Number of patients in multiple PT-INR measurement

\begin{tabular}{|c|c|c|c|c|}
\hline $\begin{array}{l}\text { Number of PT-INR } \\
\text { measurements }\end{array}$ & Number of patients & $\begin{array}{c}\text { Lab-INR } \\
(\text { mean } \pm S D)\end{array}$ & $\begin{array}{l}\text { CoaguCheck } \\
(\text { mean } \pm S D)\end{array}$ & $\begin{array}{c}\text { INRatio } \\
(\text { mean } \pm S D)\end{array}$ \\
\hline 1 & 160 & $1.9 \pm 0.4$ & $2.0 \pm 0.4$ & $2.2 \pm 0.5$ \\
\hline 2 & 138 & $1.9 \pm 0.4$ & $2.0 \pm 0.4$ & $2.3 \pm 0.6$ \\
\hline 3 & 36 & $2.0 \pm 0.4$ & $2.3 \pm 1.1$ & $2.5 \pm / 1.1$ \\
\hline 4 & 4 & $2.4 \pm 0.2$ & $2.4 \pm 0.2$ & $3.1 \pm 1.3$ \\
\hline 5 & 35 & $2.1 \pm 0.6$ & $2.2 \pm 0.7$ & $2.4 \pm 0.7$ \\
\hline 6 & 348 & $2.0 \pm 0.3$ & $2.0 \pm 0.3$ & $2.3 \pm 0.6$ \\
\hline 7 & 497 & $2.0 \pm 0.3$ & $2.1 \pm 0.4$ & $2.3 \pm 0.6$ \\
\hline 8 & 72 & $2.0 \pm 0.5$ & $2.2 \pm 0.8$ & $2.4 \pm / 0.9$ \\
\hline 9 & 9 & $2.3 \pm 0.7$ & $2.4 \pm 0.8$ & $2.7 \pm 0.7$ \\
\hline 10 & 0 & & & \\
\hline 11 & 22 & $2.1 \pm 0.40$ & $2.2 \pm 0.4$ & $2.4 \pm 0.5$ \\
\hline 12 & 0 & & & \\
\hline 13 & 26 & $2.1 \pm / 0.3$ & $2.22 \pm 0.3$ & $2.5 \pm 0.4$ \\
\hline
\end{tabular}

Blank cell means no measured values. 
A

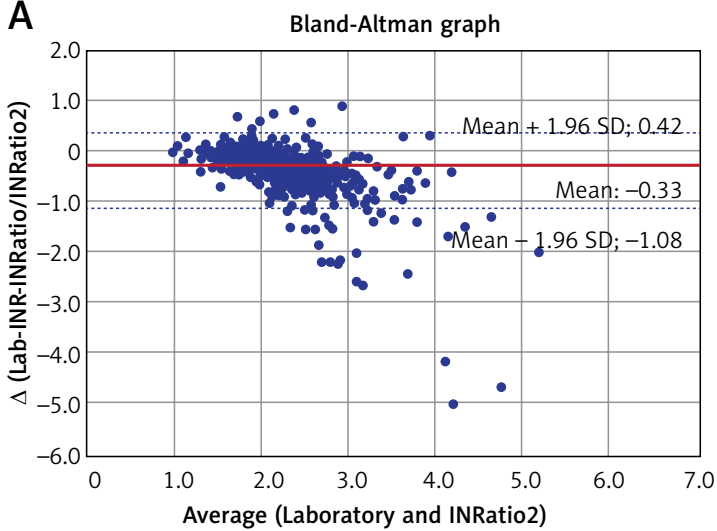

B

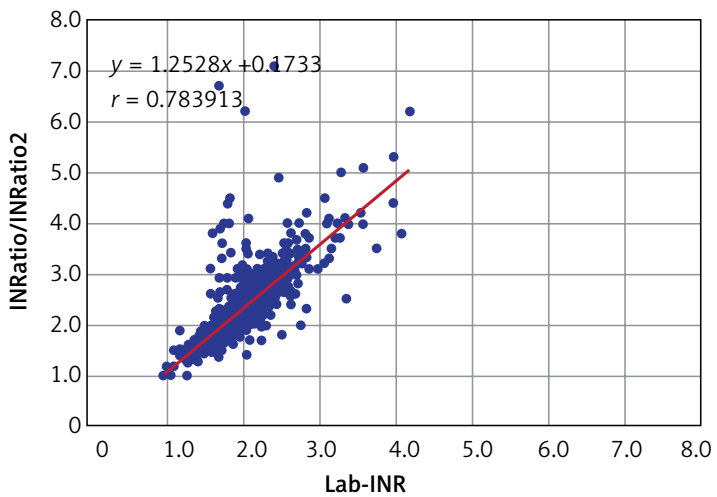

Figure 1. Comparison between PT-INR Values Measured by Traditional Plasma-Based Measurements (Lab-INR) and by Point-Of-Care Device of INRatio/INRatio2 (INRatio). A - PT-INR values measured by traditional plasma-based measurements (lab-INR) and by point-of-care device of INRatio/INRatio 2 (INRatio) as a Bland-Altman plot. The horizontal axis represent the mean of lab-INR and PT-INR measured as INRatio. The vertical axis represents the $\Delta$, which is the difference between PT-INR values measured by lab-INR and INRatio. The apparent difference becomes larger at PT-INR 2.5 or more. B - the relationship between the PT-INR values measured by lab-INR and INRatio. There was a positive correlation between the two values with a regression coefficient of 0.784

A

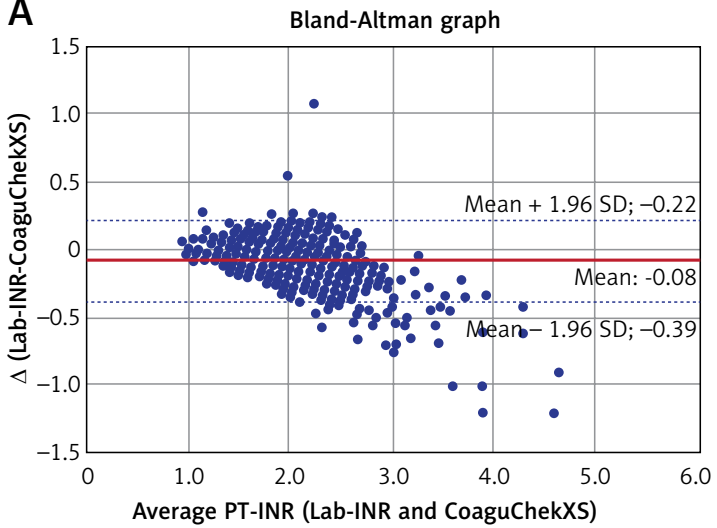

B

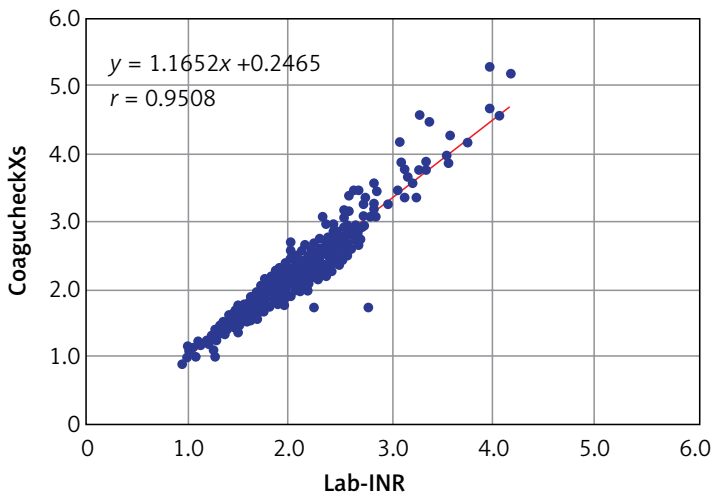

Figure 2. Comparison between PT-INR Values Measured by Traditional Plasma-Based Measurements (Lab-INR) and by Point-Of-Care Device CoaguChek XS (CoaguChek XS). A - the PT-INR values measured by traditional plasma-based measurements (lab-INR) and by point-of-care device CoaguChek XS as a Bland-Altman plot. The horizontal axis represents the mean of lab-INR and PT-INR measured by CoaguChek XS. The vertical axis represents the $\Delta$, which is the difference between PT-INR values measured by lab-INR and CoaguChek XS. The apparent difference becomes larger at PT-INR 2.5 or more. B - the relationship between the PT-INR values measured by lab-INR and CoaguChek XS. There was a positive correlation between the two values with a regression coefficient of 0.951

procedure [18]. However, it is neither easy nor convenient to separate plasma, send to a central laboratory, and wait for measured values. POC devices, enabling direct PT-INR measurements from whole blood, provide substantial advantages for daily clinical practice to reduce the risk of stroke in AF patients [19-21]. Moreover, POC devices were necessary to conduct double blind clinical trials comparing quality controlled VKAs and newly developed direct oral anticoagulants (DOACs) [12-15]. Previous publications suggest the accuracy of the POC device as compared to lab-INR in various clinical conditions [22-25]. But, the majority of the publications were limited to the use of CoaguChek XS [22-25]. Here, we confirmed that the PT-INR measured with CoaguChek XS had no difference compared to lab-INR. However, the values measured by INRatio/INRatio 2 were approximately 0.3 higher than lab-INR. Similar differences may be observed by other whole blood based POCs. Neither INRatio nor INRatio 2 is widely used in clinical practice, but they were used in some of the clinical trials such as the Rivaroxaban Once Daily Oral Direct Factor Xa Inhibition Compared with Vitamin K Antagonism for Prevention of Stroke and Embolism Trial in Atrial Fibrillation (ROCKET AF) and the J-ROCKET AF study [17, 26]. The differences are not negligible because the PTINR recommendation with the use of INRatio and INRatio 2 should be changed from 2-3 or 1.6-2.6 to $2.3-3.3$ or $1.9-2.9$. Our study raises caution for the selection and use of a POC device when considering its use in clinical trials that include controlled VKA arm(s). 
Despite wide use of PT-INR in clinical practice, PT-INR is not an accurate predictor for the onset of thrombotic/bleeding complications [27, 28]. PTINR also does not reflect the activity of vitamin $\mathrm{K}$ dependent coagulant factors precisely. Indeed, serious hemophilia B patients having a coagulation factor IX activity below $1 \%$ still have PT-INR in the normal range [29]. Practice guidelines in various regions of the world recommend a target PT-INR of $2-3$ in various pathological conditions. It is of note that the guideline recommendation is only a guide. Recently developed computer-based artificial intelligence found more precise prediction of future clinical events from serially measured PTINR [30], but a one-time measurement is still the standard.

In nature, PT-INR is calculated from the ratio of clotting time of plasma anticoagulated by relatively weak calcium ion chelating agent of citrate by addition of excess calcium ion and thromboplastin. Conceptional "thromboplastin" is identified as the complex of tissue factor [31, 32] and phospholipids [33, 34]. There are many thromboplastin agents with various activity to induce coagulation. Adjustment of measured prothrombin time ratio by the internationalized ratio is recommended. Despite adjustments, PT-INR values are known to differ substantially when using different reagents. PT-INR is one measure for evaluating the anticoagulant effect(s) of warfarin, but various values may appear with various reagents.

In December 2014, the FDA issued a recall notice for a medical device correction of the Alere INRatio Monitor System (formally known as the HemoSense INRatio device). (INRatio/INRatio2) because this POC device may provide PT-INR results which are lower than standard plasma-based laboratory INR in patients with abnormal hematocrit levels, increased fibrinogen, and so on [35]. The authors of the ROCKET-AF trials conducted comparable analysis between the 8942 of 14,236 total patients $(63 \%)$ without a recall condition and $5294(37 \%)$ in whom there was a recall condition in the ROCKET-AF trial. In both sub-groups, there was noninferiority of rivaroxaban versus warfarin for preventing stroke and systemic embolism, with similar rates of overall bleeding [35]. Similar confirmative results were published from the group of authors of the J-ROCKET AF [36]. Although lower values in INRatio/INRatio2 as compared to the labINR were cautioned by the FDA, our results here show the opposite: a higher value of PT-INR with INRatio/INRatio2. There are many potential factors influencing the PT-INR values measured with INRatio/INRatio2, not limited to the ones raised by the FDA. The important issue of note is that the values of PT-INR measured by POCs are not the same as standard lab-INR. The variability of values with various measurements is not surprising. Preven- tive effects or rivaroxaban on thrombus formation are true [1], but the physician has to be aware of the variability among values obtained by various devices [37].

Our study has a strong limitation in regard to generalization of our results to the global scale because the study was performed as a single-center registry in a single country, Japan. Standard lab-INR may differ substantially with the use of thromboplastin with different International Sensitivity Index (ISI) values. Moreover, the study was designed as a hypothesis generation study without prior prediction of necessary sample size or power calculation. The decision to use INRatio/INRatio 2 and CoaguChek XS as the devices to measure POC derived PT-INR were not made systematically by selecting from all available POC devices but were dependent upon their availability in the study clinic. Due to these strong limitations, this paper should be considered only as hypothesis generating and the findings of the variability among PT-INR values obtained by different measurement methods should be confirmed by further future studies with a larger sample size, making it possible to clarify the potential parameters influencing the values obtained by various devices.

In conclusion, our results strongly suggested the presence of variability among PT-INR values obtained by different methods.

\section{Acknowledgments}

The authors would like to express their appreciation to Ikuyo Kusano, Atsuko Seto and Namiko Takiguchi for their clerical assistance. The authors also acknowledge Dr. Shinichi Goto for correction of English grammar.

\section{Conflict of interest}

The INRatio/INRatio 2 device and test strips for study 1 were provided by Alere Medical Ltd. This device was also used as device 2 in the third study to evaluate inter-device differences. The authors $\mathrm{CK}, \mathrm{HS}$, SG have nothing to disclose. The last author, SG, acknowledges a grant-in-aid for MEXT/ JSPS KAKENHI 19H03661, AMED grant number A368TS, Bristol-Myers Squibb for independent research support project (33999603), a grant from Nakatani Foundation for Advancement of Measuring Technologies in Biomedical Engineering, and Vehicle Racing Commemorative Foundation. The last author, SG, discloses modest grant support from Sanofi, Pfizer, Bristol Myer Squibb and Ono Pharma.

\section{References \\ 1. Piotrowski R, Zaborska B, Pilichowska-Paszkiet E, et al. RIVAroxaban TWICE daily for lysis of thrombus in the}


left atrial appendage in patients with non-valvular atrial fibrillation: the RIVA-TWICE study. Arch Med Sci 2020; 16: 289-96.

2. Kjerpeseth LJ, Ellekjaer $H$, Selmer R, et al. Trends in use of warfarin and direct oral anticoagulants in atrial fibrillation in Norway, 2010 to 2015. Eur J Clin Pharmacol 2017; 73: 1417-25.

3. Camm AJ, Accetta G, Ambrosio G, et al. Evolving antithrombotic treatment patterns for patients with newly diagnosed atrial fibrillation. Heart 2017; 103: 307-14.

4. European Heart Rhythm Association; European Association for Cardio-Thoracic Surgery; Camm AJ, Kirchhof p, Lip GYH, et al. Guidelines for the management of atrial fibrillation: The Task Force for the Management of Atrial Fibrillation of the European Society of Cardiology (ESC). Eur Heart J 2010; 31: 2369-429.

5. January CT, Wann LS, Alpert JS, et al. 2014 AHA/ACC/ HRS Guideline for the Management of Patients With Atrial Fibrillation. A Report of the American College of Cardiology/American Heart Association Task Force on Practice Guidelines and the Heart Rhythm Society. J Am Coll Cardiol 2014; 64: e1-76.

6. Suzuki S, Yamashita T, Kato T, et al. Incidence of major bleeding complication of warfarin therapy in Japanese patients with atrial fibrillation. Circ J 2007; 71: 761-5.

7. Bussey HI, Force RW, Bianco TM, et al. Reliance on prothrombin time ratios causes significant errors in anticoagulation therapy. Arch Intern Med 1992; 152: 278-82.

8. Adcock DM, Duff S. Enhanced standardization of the International Normalized Ratio through the use of plasma calibrants: a concise review. Blood Coagulation Fibrinolysis 2000; 11: 583-90.

9. Tange JI, Grill D, Koch CD, et al. Local verification and assignment of mean normal prothrombin time and international sensitivity index values across various in struments: recent experience and outcome from North America. Semin Thromb Hemost 2014; 40: 115-20.

10. Sharma P, Scotland G, Criuckshank $M$, et al. The clinical effectiveness and cost-effectiveness of point-of-care tests (CoaguChek system, INRatio2 PT/INR monitor and ProTime Microcoagulation system) for the self-monitoring of the coagulation status of people receiving long-term vitamin $\mathrm{K}$ antagonist therapy, compared with standard UK practice: systematic review and economic evaluation. Health Technol Assess 2015; 19 1-172.

11. Bauman ME, Black KL, Massicotte MP, et al. Accuracy of the CoaguChek XS for point-of-care international normalized ratio (INR) measurement in children requiring warfarin. Thromb Haemost 2008; 99: 1097-103.

12. Giugliano RP, Ruff CT, Braunwald E, et al. Edoxaban versus warfarin in patients with atrial fibrillation. $N$ Engl J Med 2013; 369: 2093-104.

13. Granger CB, Alexander JH, McMurray JJV, et al. Apixaban versus warfarin in patients with atrial fibrillation. N Engl J Med 2011; 365: 981-92.

14. Patel MR, Mahaffey KW, Garg J, et al. Rivaroxaban versus warfarin in nonvalvular atrial fibrillation. $N$ Engl J Med 2011; 365: 883-91.

15. Connolly SJ, Exekowitz MD, Yusuf S, et al. Dabigatran versus warfarin in patients with atrial fibrillation. N Engl J Med 2009; 361: 1139-51.

16. Taborski U, Braun SL, Völler H. Analytical performance of the new coagulation monitoring system INRatio for the determination of INR compared with the coagulation monitor Coaguchek S and an established laboratory method. J Thromb Thrombolysis 2004; 18: 103-7.
17. Hori M, Ohashi Y, Pan G, et al. Point-of-care device for warfarin monitoring used in the J-ROCKET AF study. Circ J 2016; 80: 1488-90.

18. Woodhams BJ, Klein N, Harz D, Rose M, Ruiz JA. A study of the variability seen in the international normalized ratio obtained using different sensitivity thromboplastin reagents on different instrument types. Blood Coagul Fibrinolysis 1999; 10: 423-7.

19. Kotlęga D, Gołąb-Janowska M, Meller A, et al. Beneficial effects of pre-stroke statins use in cardioembolic stroke patients with atrial fibrillation: a hospital-based retrospective analysis. Arch Med Sci 2019; 15: 385-92.

20. Demir GG, Güneş HM, Seker M, et al. Is the presence of left atrial diverticulum associated with recurrence in patients undergoing catheter ablation for atrial fibrillation? Arch Med Sci Atheroscler Dis 2019; 4: e25-31.

21. Negreva M, Prodanova K, Vitlianova K, Madjova C. Paroxysmal atrial fibrillation: changes in factor VIII and von Willebrand factor impose early hypercoagulability. Arch Med Sci Atheroscler Dis 2020; 5: 140-7.

22. Bauman ME, Black KL, Massicotte MP, et al. Accuracy of the CoaguChek XS for point-of-care international normalized ratio (INR) measurement in children requiring warfarin. Thrombosis Haemostasis 2008; 99: 1097-103.

23. Lakshmy R, Kumar AS. Comparative evaluation of point of care coagulation monitoring by coaguchek XS-comparison with standard laboratory method. Indian J Thor Cardiovasc Surg 2010; 26: 125-8.

24. Oral Anticoagulation Monitoring Study Group. Point-ofcare prothrombin time measurement for professional and patient self-testing use: a multicenter clinical experience. Am J Clin Pathol 2001; 115: 288-96.

25. Bauman ME, Black KL, Massicotte MP, et al. Accuracy of the CoaguChek XS® for POC INR in warfarinised children and adults with ventricular assist devices. Thrombosis Haemostasis 2013; 110: 616-7.

26. Patel MR, Hellkamp AS, Fox KA. Point-of-care warfarin monitoring in the ROCKET AF Trial. N Engl J Med 2016; 375: 390-1.

27. Wysowski DK, Nourjah P, Swartz L. Bleeding complications with warfarin use: a prevalent adverse effect resulting in regulatory action. Arch Intern Med 2007; 167 1414-9.

28. Palareti G, leali N, Coccheri S, et al. Bleeding complications of oral anticoagulant treatment: an inception-cohort, prospective collaborative study (ISCOAT). Italian Study on Complications of Oral Anticoagulant Therapy. Lancet 1996; 348: 423-8.

29. Dargaud Y, Hoffman M, lefrapper L, et al. Bleeding risk in warfarinized patients with a therapeutic international normalized ratio: the effect of low factor IX levels. J Thromb Haemost 2013; 11: 1043-52.

30. Goto S, Goto S, Pieper K, et al. New Al prediction model using serial PT-INR measurements in AF ptients on VKAs: GARFIELD-AF. Eur Heart J Cardiovasc Pharmacother 2019; pvz076. doi: 10.1093/ehjcvp/pvz076.

31. Tripodi A, Arbini A, Chantarangkul V, Mannucci PM. Recombinant tissue factor as substitute for conventional thromboplastin in the prothrombin time test. Thromb Haemost 1992; 67: 42-5.

32. Bjorklid E, Storm E, Prydz H. The protein component of human brain thromboplastin. Biochem Biophys Res Commun 1973; 55: 969-76.

33. Liu DT, McCoy LE. Phospholipid requirements of tissue thromboplastin in blood coagulation. Thromb Res 1975; 7: 213-21. 
34. Smith SA, Comp PC, Morrissey JH. Phospholipid composition controls thromboplastin sensitivity to individual clotting factors. J Thromb Haemost 2006; 4: 820-7.

35. Patel MR, Hellkamp AS, Fox KAA. Point-of-care warfarin monitoring in the ROCKET AF trial. N Engl J Med 2016; 374: 785-8.

36. On behalf of the J-ROCKET AF Study Investigators, Hori M, Ohashi Y, Pan G, Kato M, Kajikawa M. Point of-Care Device for Warfarin Monitoring Used in the J-ROCKET AF Study. Circulation J 2016; 80: 1488-90.

37. Cohen D. Rivaroxaban: can we trust the evidence? BM 2016; 352: i575. 